\title{
INDUCED PLURIPOTENT STEM CELLS AND THEIR IMPLICATION FOR BIOMEDICAL SCIENCES
}

\author{
${ }^{1}$ Maria Csobonyeiova, ${ }^{1}$ Stefan Polak and ${ }^{2}$ Lubos Danisovic \\ ${ }^{1}$ Institute of Histology and Embryology, \\ ${ }^{2}$ Institute of Medical Biology, Genetics and Clinical Genetics, \\ Faculty of Medicine, Comenius University, Bratislava, Slovakia
}

Received 2013-09-05, Revised 2013-09-26; Accepted 2013-10-03

\begin{abstract}
The groundbreaking discovery, that somatic mammalian cells can be epigenetically reprogrammed to a pluripotent state through the exogenous expression of the transcription factors Oct4, Sox2, Klf4 and c-myc, has yielded a new cell type for potential application in regenerative medicine, the induced Pluripotent Stem Cells (iPSCs). Since the first demonstration of creating iPSCs in 2006 great efforts have been made into improving iPS cell generation methods and understanding the reprogramming mechanism as well as the nature of iPSCs. The iPSCs technique makes it possible to produce patient-specific pluripotent stem cells for transplantation therapy without immune rejection. However, some restriction still remain, including viral vector integration into the genome, the existence of exogenous oncogenic factors and low induction efficiency. In this review we discuss recent advances in methods of generating safer iPSCs lines and their possible use for biomedical applications.
\end{abstract}

Keywords: Induced Pluripotent Stem Cells, Reprogramming, Gene Therapy, Regenerative Medicine

\section{INTRODUCTION}

The ability to reprogram a somatic cell into a pluripotent cell has been a goal of regenerative research for many decades. Initial attempts to reprogram cells involved transplanting a somatic nucleus into an enucleated oocyte, called Somatic Cell Nuclear Transfer (SCNT) (Lenger, 2010; Nsair and MacLellan, 2011). The procedure was first successfully accomplished in mammals in 1996 with the birth of a cloned lamb. This discovery caused a paradigm shift in developmental biology. However, the process was very inefficient and the reprogramming incomplete leading to Dolly's premature death and early onset of a number of degenerative disease (Kang et al., 2010; Patel and Yang, 2010). This reprogramming method also has proved successful for several species, but there are technical issues in applying this approach to human cells (Feng et al., 2009). The other major impediment of nuclear transfer-derived Embryonic Stem Cells (ESCs) based therapies in humans involves the moral and ethical dilemma surrounding the requisite blastocyst destruction and oocyte donation necessary to generate patient-specific pluripotent stem cells (Lenger, 2010). Reprogramming of somatic cell by nuclear transfer indicates that unfertilized eggs and ESCs contain factors that can induce pluripotency. Therefore, nuclear reprogramming studies using SCNT have demonstrated that transcriptional factors are essential for the reprogramming of terminally differentiated cells.

Based on this hypothesis, 24 different candidate factors were examined for their ability to induce pluripotency (Ma et al., 2008; Oh et al., 2011). In 2006 Yanamaka's group demonstrated that viral transduction of murine fibroblasts with only four transcription factors, Oct4, Sox2, Klf4 and c-Myc, could reprogram these cells back to an undifferentiated embryonic state. Human iPSCs were first generated in the same lab by transducing adult human dermal fibroblasts with viral vectors carrying the key pluripotency genes (Yamanaka factors). Thomson's group independently published a similar screening strategy using 
only human materials and identified that Oct4, Sox2, Nanog and Lin 28 were sufficient to reprogram human cells. Since then, alternative strategies for generating iPSCs have been developed. These completely reprogrammed cells are similar to ESCs in capacity for self-renewal in vitro, pluripotency, cell morphology, patterns of epigenetic changes, capability to form embryoid bodies, teratoma, viable chimeras and moreover, murine iPSCs, when they are injected into tetraploid blastocyst, can developed into whole organism. On the other hand, several groups have compared the molecular signatures of iPSCs and ESCs in their undifferentiated state. One study showed that while iPSCs were more similar to ESCs than to the paternal somatic cells, they still possessed a distinct gene expression signature. Other great advantage is that iPSCs avoid ethical issues because they do not involve the use of embryonic or fetal material (Cui et al., 2009; Herberts et al., 2011; Li et al., 2010; Medvedev et al., 2010; Nsair and MacLellan, 2011; Takahashi and Yamanaka, 2006). Today, these cells have become the major tools in advancement of personalized medicine (Ferreira and Mostajo-Radji, 2013).

\section{1. iPSCs Generation by Viral Vector}

Several methods have been used thus far to produce iPSCs (Fig. 1), which involve the transfer of genes into the target cell (Deng, 2010). The system of gene delivery system is a key aspect for iPS cell generation. Many researches in this field use viral or nonviral methods to reprogram mature cell and some groups have tried to use nongenetic materials for the generation of efficient and safe iPSCs. However, there are still a number of problems related to current reprogramming methods (Oh et al., 2011).

Most of the iPSCs lines established to date have been generated using integrating retroviral and lentiviral vectors to deliver reprogramming factors. The major advantage of using retroviral vectors is that expression of the pluripotency factors is driven by the retroviral LTR (long terminal repeat). The retroviral LTR becomes silenced in pluripotent stem cells, so upon correct reprogramming of the fibroblast and expression of the endogenous pluripotency genes, the exogenous reprogramming factors are silenced. The potential for oncogenesis due to insertional mutagenesis that is inherent to stable genomic integration has been identified as a limitation. However, it is important to recognize that distinct advantages of the retroviral-based vector systems enabled critical insight into the fundamental mechanisms of nuclear reprogramming (Seifinejad et al., 2010).

Later generations of iPSCs were produced using lentiviruses. They are less mutagenic than retroviruses but are also not a perfect alternative. Unlike retroviruses, lentiviruses infect both dividing and non-dividing cells, hence could vastly improve the rates of cell transduction and the production of lentiviruses is not overly complicated, therefore, very efficient, stable, reproducible gene expression could be achieved and wide variety of cells could be transduced. Although retrovirally-transduced genes are generally subject to epigenetic silencing during the process of iPSCs induction, reactivation of these genes in vivo may lead to a higher risk of tumor incidence and malignant progression. While retro- and lentiviral integration is thought to occur randomly in the genome, certain events may cause activation of nearby oncogene or inactivation of a tumor suppresor gene (Duinsbergen et al., 2009; Gardlik, 2012; Wong and Chiu, 2011).

The attempts to make iPSCs more therapeutically applicable have led to the pursuit of nonintegrating virus vectors. Adenovirus, which is a nonintegrating vector and remains as an epichromosomal form in cells, offers a flexible platform. However, adenovirus is generally rather poor at gene transfer. It is also very difficult to control the level of gene expression infected cell (Lai et al., 2011; Patel and Yang, 2010).

Another viral vector system without genomic integration is Sendaiviral (Sendaivirus) vector, which is a RNA virus that replicates its genome exclusively in the cytoplasm, efficiently generated iPSCs from human somatic cells (Macarthur et al., 2012; Oh et al., 2011).

\section{2. iPSCs Generation by Non-Viral Vector}

The success of the non-integrating vector with transient gene expression to generate iPSCs has provided an opportunity to potentially develop a non-viral delivery strategy, which is safe, cost-effective, easier to manufacture and manipulate. Non-viral delivery system includes episomal plasmide transfection, minicirle plasmids, piggyBac transposition system, protein transduction and most recently, repeated administration of synthetic mRNA and using of small molecules (Huang and $\mathrm{Wu}, 2012$; Nie et al., 2012; Wong and Chiu, 2011).

Plasmide transfection usually uses plasmids carrying the reprogramming genes encapsulated by lipid or cationic polymers and subsequently trasfected into the cells to be reprogrammed. Episomal plasmid vectors are able to replicate themselves autonomously as extrachromosomal element. They also exhibit prolonged expression of the reprogramming genes in target cells. Nevertheless, the reprogramming efficiency of this approach was extremely low (Lai et al., 2011; Medvedev et al., 2010; Yu et al., 2011). 


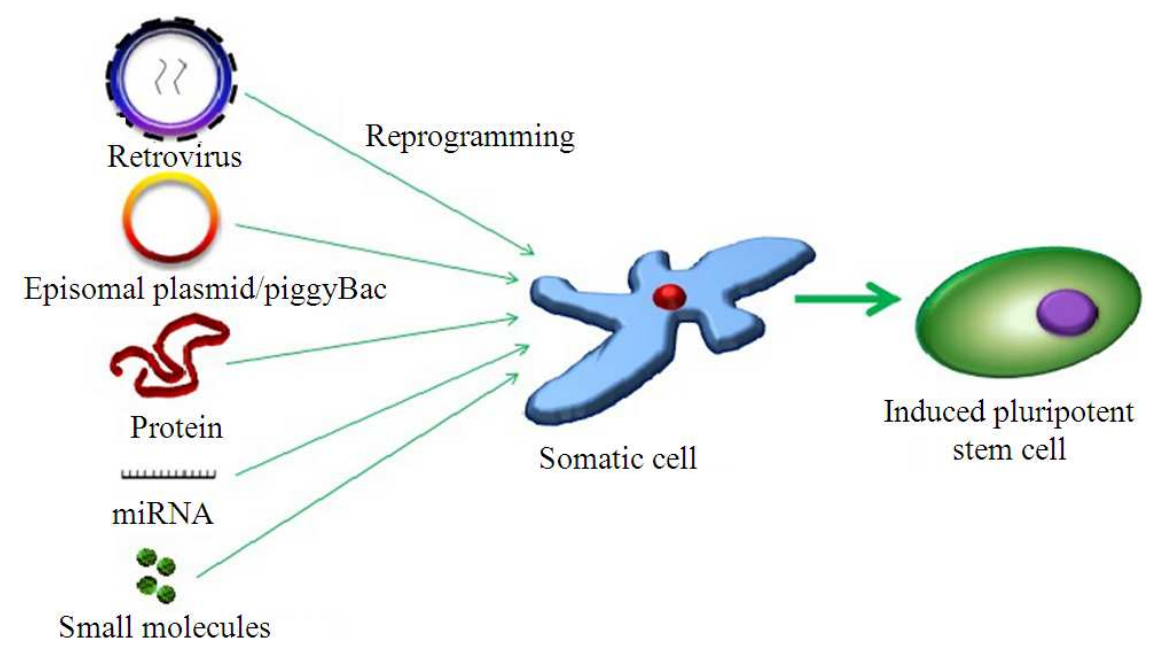

Fig. 1. Reprogramming strategies used to induce pluripotent stem cells from adult somatic cells

The piggyBac system-is promising system used for iPSCs production without any modification of the host genome based on DNA transposons. The transposon includes a mobile genetic element that can be used to integrate transgenes. Successful transposon-based reprogramming of fibroblasts to iPSCs represents a significant advance in current methods of transgene delivery. This system couples enzymatic cleavage with sequence specific recognition using transpon/transponase interaction to ensure high efficiency removal of flanked DNA without footprint. It permits technical simplification and improved accessibility of reprogramming methodology by making use of effortless plasmid DNA preparation and commercial transfection products for delivery. Also the range of somatic cell types that could be used for reprogramming is not limited by a decreased susceptibility to viral infection. Importantly, this study also demonstrated that continued expression of exogenous reprogramming factors is not required for in vitro reprogramming (Seifinejad et al., 2010; Woltjen et al., 2009). However, despite the high efficiency of exogenous DNA excision from the genom by this system, the removal of a large number of transposon copies is hardly achievable (Medvedev et al., 2010).

Direct protein transduction system represent significant advance in generating iPSCs and has several major advantages. The observation that some exogenous proteins are capable of being taken up by the cell has resulted in the identification of protein transduction domains, also called "transduction peptides" or "cell-penetrating peptides". When fused to other proteins, these peptides allow the cargo protein to enter the cell, although at different efficiencies (Seifinejad et al., 2010). This method eliminates limitation that may be caused by viral or any other DNA-based reprogramming methods, it means the potential risks associated with chromosomal integration and mutation. Zhou et al. (2009) demonstrated that protein-induced Pluripotent Stem Cells (p-hiPSCs) can long-term self-renew and are pluripotent in vitro and in vivo. These iPSCs exhibited similarity to ESCs in morphology, proliferation and expression of characteristic pluripotency markers. The mouse iPSCs were generated using recombinant proteins of reprogramming factors which were produced in bacteria. However, iPSCs derivation required the addition of the histone deacetylase inhibitor valopronic acid. Their study first demonstrated that somatic cells can be fully reprogrammed into pluripotent stem cells by direct delivery of recombinant reprogramming proteins. However, the generation of p-hiPSCs is very inefficient and requires further optimization. The efficiency of production is around $0,001 \%$, which is one order lower than by using retroviral vectors. In particular, the concentration of the individual factors needs to be calibrated to approximate normal endogenous level (Kim et al., 2009; Lai et al., 2011; Wong and Chiu, 2011).

Yakubov et al. (2010) reported the use of in vitroproduced mRNA encoding for Oct4, Lin28, Sox2 and Nanog for reprogramming of human foreskin fibroblasts to generate RNA-produced iPSCs (RiPSCs). This procedure also avoids any DNA integration events and the associated genomic damage. The procedure of RNA preparation in vitro is simple and easy to scale up 
and transfection is very efficient. The production of RiPSCs by RNA overcomes an important hurdle on the way to personalized cell therapy.

Small molecules-another alternative approach to safety improve the reprogramming process for the generation of iPSCs is to use a cocktail of small molecules that are linked with epigenetic modifiers and major signaling pathways. Notably, some of these molecules can also replace individual reprogramming factors, raising the possibility of deriving iPSCs solely with chemicals. These molecules are inhibitors of Histone De A Cetylases (HDACs)-Val Proic Acid (VPA), Tricho Statin A (TSA), Histone De Methylases (HDMs) and Histone Methyl Transferases (HMTs), which regulate chromatin remodeling and act as major players in building up the epigenetic landscape. HDACc inhibitors significantly were shown to improve reprogramming efficiency. Also other small molecules have been reported to be able to replace some transcription factors. For example, a $\mathrm{G}_{9 \mathrm{a}}$ inhibitor, BIX $_{01294}$, was reported to induce iPS cells from neural stem cells, in place of Oct4. In addition, a Transforming Growth Factor (TGF)- $\beta$ inhibitor could replace Sox 2 during iPSCs generation. Vitamin $\mathrm{C}$ also improves the efficiency of reprogramming in both mouse and human somatic cells and promotes the transition of partially reprogrammed iPSCs to fully reprogrammed state likely by reducing reactive oxidant species and senescence.

Because small molecules provide several distinct advantages in controlling protein fusion, they have attracted much interest for control reprogramming toward a faster and more efficient process. Chemical strategies are being used to find additional small molecules that may ultimately allow reprogramming of lineage-restricted cells to pluripotent state in a completely chemically defined condition. However, it should be noted that chemical substitution of a reprogramming factors is, in most cases, associated with a significant decrease in the number of iPSCs clones generated, indicating that no single chemical compound is able to entirely replace the function of a transcription factor (Feng et al., 2009; Li et al., 2011; Lu et al., 2013; Nie et al., 2012; Stadtfeld and Hochedlinger, 2010; Oh et al., 2011; Shi et al., 2008).

\subsection{Applications of Human iPSCs}

Human iPSCs might be used to treat degenerative and genetic diseases and to study diseases mechanism at the individual patient level (Cui et al., 2009). They could generate a limitless source for tissue engineering and regenerative medicine applications (Fig. 2). Another key advantage is the possibility of repairing disease-causing mutation by homologous recombination, a technology that has been used with limited success in adult stem cells because of notorious difficulties in growing them outside the body. Human iPSCs could have also significant impact on drug development and toxicity tests to replace and refine animal experiments and test (Martinez-Fernandez et al., 2010; Pappas and Yang, 2008; Stadtfeld and Hochedlinger, 2010; Vitale et al., 2011).

Recently, there have been great advancements in the study of iPSCs in the field of regenerative medicine. Organ transplantation among nonrelated individuals is complicated by the limited availability of matched tissues and requirement for life-long treatment with immunosupresive drugs that can have serious side effects. Somatic cells isolated from a patient may be reprogrammed to pluripotent stem cells and then theoretically could be used to replace diseased cells in the same patient avoiding the problem of histocompatibility or immune rejection. Many human diseases, such as myocardial infarction, diabetes, retinal degeneration and spinal cord injury, occur because of cell loss, degeneration and injury. Theoretically, with the transplantation of specific cells created from autologus iPS cells, the cells that lacking can be replenished and replace by cells with the defects corrected, thereby relieving a patient's symptoms. The mostly use somatic cells are fibroblasts, but different groups generated also iPS cells from other somatic cells providing evidence that is possible to reprogram cells of different origins (Deng, 2010; Ebben et al., 2011; Patel and Yang, 2010; Uemura et al., 2012; Vitale et al., 2011; Walia et al., 2012; Wong and Chiu, 2011; Zeng and Zhou, 2011). Other sources of iPSCs that can be easily reprogrammed are human keratinocytes (Aasent et al., 2008; Petit et al., 2012), oral mucosa fibroblasts (Miyoshi et al., 2010), dermal papilla cells (Tsai et al., 2010), pancreatic beta cells (Stadtfeld et al., 2008), neural stem cells (Kim et al., 2009), mature B lymphocytes (Hanna et al., 2008), liver and stomach cells (Aoi et al., 2008) and cord blood cells (Cai et al., 2010; Takenaka et al., 2009). For example, human keratinocytes have been shown to be 100 -fold more efficient and 2-fold faster than human fibroblasts (Aasent et al., 2008). The goal is to find the most accessible, efficient and safest cell to reprogram to future clinical applications. 


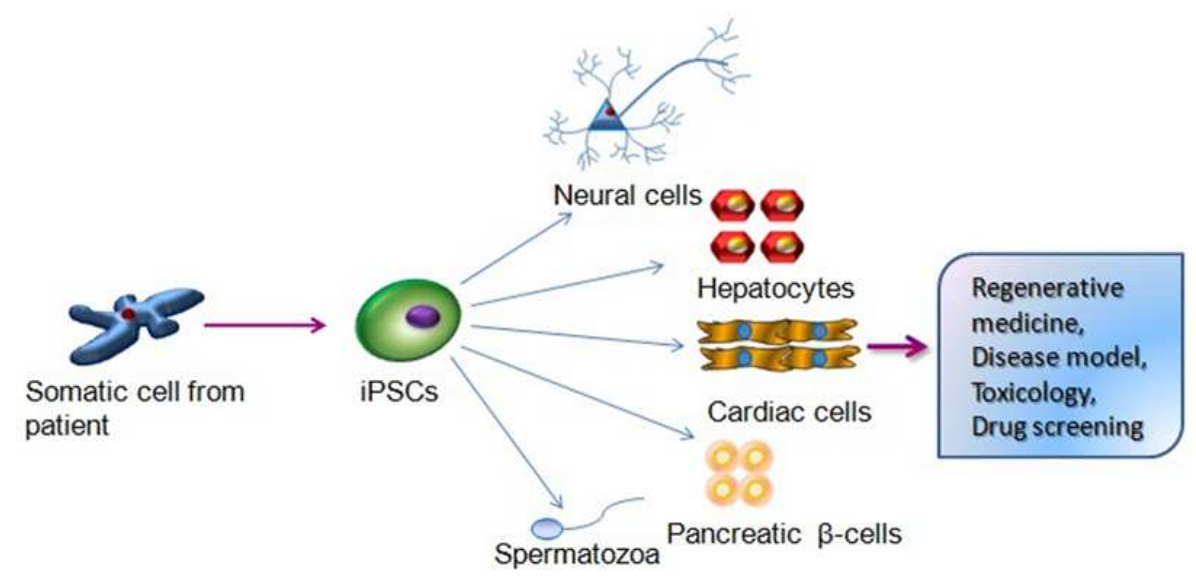

Fig. 2. Applications of iPSCs technology

The potential use of iPSCs as treatments for various disorders has been proposed and tested on in vitro and/or in vivo animal models with promising results (Patel and Yang, 2010). Proof of principle for iPSCsbased therapy was first demonstrated in mice. iPSCs were generated from tail-tip fibroblasts of humanized sickle cell anemia mice. After the sickle cell gene mutation in the generated iPSCs was corrected via homologous recombination, the cells were directed to differentiate into hematopoetic progenitor cells in vitro. After they were transplanted into the affected donor mice, resulting in restoration of the functional hemoglobin protein in the bloodstream and restoring disease parameters such as red blood cell count, hematocrit, weight and breath rate to normal values. Also kidney defects due to the red blood cell destruction in renal tubules with reduction in renal blood flow were rescued upon iPSCs therapy (Cui et al., 2009; Hanna et al., 2007). Thus, the first therapeutic application of iPSCs technology illustrated the advantages of both regeneration of degenerative disease as well as gene-specific correction of an inheritable effect (Nelson et al., 2010).

For example, of particular interest are iPSCs derived from patients suffering from neurodegenerative diseases. These iPSCs lines can be differentiated in vitro into the affected neuronal cell type (Lenger, 2010). iPSCs have been generated from patients with amyotryophic lateral sclerosis and they could be differentiated into motor neurons. Greater progress has been made in generating enriched population of ventral midbrain dopaminergic neurons that are relevant for Parkinson disease (Marchetto et al., 2011).
It was also demonstrated that murine iPSCs have ability to differentiate into mesenchymal cells like cells of bone, cartilage and fat in vitro and maintain an osteoblast phenotype on a scaffold in vitro and in vivo. The maintenance of this stable osteoblast phenotype spotlights these cells as a viable source for clinical cellbased therapy to treat musculoskeletal disease (Bilousova et al., 2011).

First endodermal-derived cells differentiated form iPS cells were hepatocytes, which could potentially revolutionize hepatology with respect to the study of hepatitis B and C viruses, alcohol-induced cirrhosis and congenital liver diseases (Gallicano and Mishra, 2010).

Other potential application in near future can be use of iPSCs derived spermatozoa to treat infertile couples, where male suffer from Non-Obstructive Azoospermia (NOA) (caused by testis failure or impaired spermatogenesis). These patient specific iPSCs derived spermatozoa can help patient create their own genetic offspring via ICSI, they also allow the development of novel reproductive engineering afpproaches and advanced studies on pathogenesis and treatment of fertility (Yao et al., 2011).

\subsection{Future Perspectives}

Despite success in animal models, iPSCs technology is not yet ready for transplanting cells into patients. The main issue is safety concerns, iPSCs, like ES cells, tend to form teratomas and current differentiation protocols cannot efficiently eliminate residual undifferentiated cells. The researches still do not know for each iPSCs clone whether nuclear reprogramming is complete. Aberrant reprogramming may result in an impaired 
ability to differentiate and may increase the risk of immature teratoma formation after direct differentiation. Most patient-specific iPSCs have been generated with integrating vectors, which may not get silenced efficiently or could disrupt endogenous genes, which also pose potential impediments for the use of human iPSCs in cell therapy. For diseases that require gene targeting to repair mutant alleles, more efficient targeting strategies need to be developed. However, the recent study showed that even patient's own iPSCs may induce immune rejection. Moreover, when viral vectors are used to engineer the expression vectors for the various methods for genetic repair, immune rejection may be developed towards the viral vector (Cha and Hwang, 2012; Pawitan, 2012; Stadtfeld and Hochedlinger, 2010; Yamanaka, 2009).

Extensive research has aimed at the generation of human iPSCs using methods that avoid the use of retroviruses and oncogenes. However, all such methods have been found to reprogram cells at low efficiency. To able to reproducibly and efficiently generate iPSCs will require in-depth understanding of the reprogramming process. Because reprogramming is in a sense the opposite of development, involving a return from the adult to embryonic status, it is possible that deeper understanding of developmental pathways may provide further insights into the reprogramming process (Li et al., 2010).

Another essential prerequisite for future widespread applications of human iPSCs is the development of efficient cryopreservation methods to facilitate cell banking. Importantly, a simple and effective cryopreservation procedure has been established for single dissociated human iPSCs in feeder-free culture using a Rho kinase inhibitor (Mollamohammadi et al., 2009).

\section{CONCLUSION}

In addition, before using iPSCs for clinical purposes, however, a few issues need to be addressed. Safety remains a critical consideration where stem cells are concerned: the long term effects of the reprogramming process on cells and their progeny must be studied very carefully. The risk of tumor formation after iPSCs treatment needs to be minimized by: reducing genetic disruption to the cell caused by reprogramming using strategies, avoiding prolonged expression of the oncogenic reprogramming factors, ensuring completion of reprogramming process and confirming differentiation capacity of iPSCs, as partial or aberrant reprogramming might be conducive to teratoma formation (Wong and
Chiu, 2011). For human iPSCs to be therapeutically effective in transplantation it needs to be also shown that their progeny will function normally in the intended site for significant periods of time, which will require extensive testing first in animals and then in appropriately designed clinical trials (Sipp, 2010).

\section{ACKNOWLEDGEMENT}

This study was supported by grant of Comenius University No. UK/197/2013.

\section{REFERENCES}

Aasent, T., A. Raya, M.J. Barrero, E. Garreta and A. Consiglio et al., 2008. Efficient and rapid generation of induced pluripotent stem cells from human keratinocytes. Nat. Biotechnol., 26: 1276-1284. DOI: 10.1038/nbt.1503

Aoi, T., K. Yae, M. Nakagawa, T. Ichisaka and K. Okita, et al., 2008. Generation of pluripotent stem cells from adult mouse liver and stomach cells. Science, 321: 699-702. DOI: 10.1126/science.1154884

Bilousova, G., D.H. Jun, K.B. King, S. De Langhe and W. Chick et al., 2011. Osteoblasts derived from induced pluripotent stem cells form calcified structures in scaffolds both in vitro and in vivo. Stem Cells, 29: 206-216. DOI: 10.1002/stem.566

Cai, J., W. Li, H. Su, D. Qin and J. Yang et al., 2010. Generation of human induced pluripotent stem cells from umbilical cord matrix and amniotic membrane mesenchymal cells. J. Biol. Chem., 285: $11227-$ 11234. DOI: $10.1074 /$ jbc.M109.086389

Cha, H.J. and E.S. Hwang, 2012. Current status of biology, bioengineering and therapeutic potential of stem cells. Arch. Pharm. Res., 35: 193-196. DOI: 10.1007/s12272-012-0200-1

Cui, C.H., L. Rao, L. Cheng and L. Xiao, 2009. Generation and application of human iPS cells. Chin. Sci. Bull., 54: 9-13. DOI: 10.1007/s11434008-0579-8

Deng, W., 2010. Induced pluripotent stem cells: Paths to new medicines. EMBRO Rep., 11: 161-165. DOI: 10.1038/embor.2010.15

Duinsbergen, D., D. Salvatori, M. Eriksson and H. Mikkers, 2009. Tumors originating from induced pluripotent stem cells and methods for their prevention. Ann. N. Y. Acad. Sci., 1176: 197-204. PMID: 19796248 
Ebben, J., M. Zorniak, P. Clark and J. Kuo, 2011. Introduction to induced pluripotent stem cells: Advancing the potential for personalized medicine. World Neurosurg., 76: 270-275. DOI: 10.1016/j.wneu.2010.12.055

Feng, B., J.H. Ng, J. CH. D. Heng and H.H. Ng, 2009. Molecules that promote or enhance reprogramming of somatic cells to induced pluripotent stem cells. Cell Stem. Cell, 4: 301-312. DOI: 10.1016/j.stem.2009.03.005

Ferreira, L.M. and M.A. Mostajo-Radji, 2013. How induced pluripotent stem cells are redefining personalized medicine. GENE, 520: 1-6. DOI: 10.1016/j.gene.2013.02.037

Gallicano, G.I. and L. Mishra, 2010. Hepatocytes from induced pluripotent stem cells: A giant leap forward for hepatology. Hepatology, 51: 297-305. DOI: 10.1002/hep. 23474

Gardlik, R., 2012. Inducing pluripotency using in vivo gene therapy. Med. Hypoth., 79: 197-201. DOI: 10.1016/j.mehy.2012.04.034

Hanna, J., M. Werning, S. Markoulaki, S. Sun and A. Meissner et al., 2007. Treatment of sickle cell anemia mouse model with iPS cells generated from autologous skin. Science, 318: 1920-1923. DOI: 10.1126/science. 1152092

Hanna, J., S. Markoulaki, P. Schorderet, B.Carey and B. Beard et al., 2008. Direct reprogramming of terminally differentiated mature B lymphocytes to pluripotency. Cell, 133: 250-264. DOI: 10.1016/j.cell.2008.03.028

Herberts, C.A., M.S.G. Kwa and H.P.H. Hermsen, 2011. Risk factors in the development of stem cell therapy. J. Transl. Med., 9: 29-44. DOI: 10.1186/1479-58769-29

Huang, C.H. and J.C. Wu, 2012. Epigenetic modulations of induced pluripotent stem cells: Novel therapies and disease models. Drug Discovery Today. Dis. Models, 9: 153-160. DOI: 10.1016/j.ddmod.2012.02.004

Kang, L., Z. Kou, Y. Zhang and S. Gao, 2010. Induced pluripotent stem cells (iPSCs)-a new era of reprogramming. J. Genet. Genomics, 37: 415-421. DOI: $10.1016 / \mathrm{S} 1673-8527(09) 60060-6$

Kim, D., C.H. Kim, J. Moon, Y. Chung and M. Chang et al., 2009. Generation of human induced pluripotent stem cells by direct delivery of reprogramming proteins. Cell Stem Cell, 4: 472-476. DOI: 10.1016/j.stem.2009.05.005
Lai, M.I., W.Y. Wendy-Yeo, R. Ramasamy, N. Nordin and R. Rosly et al., 2011. Advancements in reprogramming strategies for the generation of induced pluripotent stem cells. J. Assist. Reprod. Genet., 28: 291-301. DOI: 10.1007/s10815-0119552-6

Lenger, C.J., 2010. IPS cell technology in regenerative medicine. Ann. N. Y. Acad. Sci., 1192: 38-40. DOI: 10.1111/j.1749-6632.2009.05213.x

Li, M., M. Chen, W. Han and X. Fu, 2010. How far are induced pluripotent stem cells from the clinic? Ageing Res. Rev., 9: 257-264. DOI: 10.1016/j.arr.2010.03.001

Li, Y., Q. Zhang, X. Yin, W. Yang and Y. Du et al., 2011. Generation of iPSCs from mouse fibroblasts with a single gene, Oct 4 and small molecules. Cell Res., 21: 196-204. DOI: 10.1038/cr.2010.142

Lu, J., X. Kong, CH. Luo and K.K. Li, 2013. Application of epigenome-modifying small molecules in induced pluripotent stem cells. Med. Res. Rev., 33: 790-882. DOI: 10.1002/med.21265

Ma, M., J. Sha, Z. Zhou, Q. Zhou and Q. Li, 2008. Generation of patient-specific pluripotent stem cells and directed differentiation of embryonic stem cells for regenerative medicine. J. Nanjing Med. Univ., 22: 135-142. DOI: 10.1016/S10074376(08)60052-0

Macarthur, C.C., A. Fontes, N. Ravinder, D. Kuninger and J. Kaur et al., 2012. Generation of humanInduced pluripotent stem cells by a nonintegrating RNA sendai virus vector in feeder-free or xeno-free conditions. Stem Cell Int., 2012: 1-9. DOI: 10.1155/2012/564612

Marchetto, M.C., K.J. Brennand, L.F. Boyer and F.H. Gage, 2011. Induced Pluripotent Stem Cells (iPSCs) and neurological disease modeling: Progress and promises. Hum. Mol. Genet., 20: 109-115. DOI: 10.1093/hmg/ddr336

Martinez-Fernandez, A., T. Nelson, Y. Ikeda, A. Terzic. 2010. c-MYC-independent nuclear reprogramming favors cardiogenic potential of induced pluripotent stem cells. J. Cardiovasc. Trans. Res., 3: 13-23. DOI: $10.1007 / \mathrm{s} 12265-009-9150-5$

Medvedev, S.P., A.I. Shevchenko and S.M. Zakian, 2010. Induced pluripotent stem cells: Problems and advantages when applying them in regenerative medicine. Acta Nat., 2: 18-27. PMD: 22649638 
Miyoshi, K., D. Tsuji, K. Kudoch, K. Satomura and T. Muto et al., 2010. Generation of human induced pluripotent stem cells from oral mucosa. J. Biosci. Bioeng., $\quad$ 110: 345-350. DOI: 10.1016/j.jbiosc.2010.03.004

Mollamohammadi, S., A. Taei, M. Pakzad, M. Totonchi and A. Seifinejad et al., 2009. A simple and efficient cryopreservation method for feederfree dissociated human induced pluripotent stem cells and human embryonic stem cells. Hum. Reprod., 24: 2468-2476. DOI: 10.1093/humrep/dep244

Nelson, T.J., A. Martinez-Fernandez, S. Yamada, C. Perez-Terzic and Y. Ikeda et al., 2010. Repair of acute myocardial infarction by human stemness factors induced pluripotent stem cells. Circulation. 120: 408-416. DOI: 10.1161/CIRCULATIONAHA.109.865154

Nie, B., H. Wang, T. Laurent and S. Ding, 2012. Cellular reprogramming: A small molecule perspective. Curr. Opinion Cell Biol., 24: 1-9. DOI: 10.1016/j.ceb.2012.08.010

Nsair, A. and W.R. MacLellan, 2011. Induced pluripotent stem cells for regenerative cardiovascular therapies and biomedical discovery. Adv. Drug Deliv. Rev., 63: 324-330. DOI: 10.1016/j.addr.2011.01.013

Oh, S.I., C.K. Lee, K.J. Cho, K.O. Lee and S.G. Cho et al., 2011. Technological progress in generation of induced pluripotent stem cells for clinical applications. Scientific World J., 2012: 1-10. DOI: 10.1100/2012/417809

Pappas, J.J. and P.C. Yang, 2008. Human ESC vs. iPSCPros and Cons. J. Cardiovasc. Trans. Res., 1: 96-99. DOI: $10.1007 / \mathrm{s} 12265-008-9032-2$

Patel, M. and S. Yang, 2010. Advances in reprogramming somatic cells to induced pluripotent stem cells. Stem Cell Rev. Rep., 6: 367-380. DOI: 10.1007/s12015-010-9123-8

Pawitan, J.A., 2012. Prospect of induced pluripotent stem cell genetic repair to cure genetic diseases. Stem Cells Int., 2012: 1-7. DOI: $10.1155 / 2012 / 498197$

Petit, I., N.S. Kesner, R. Karry, O. Robicsek and E. Aberdam et al., 2012. Induced pluripotent stem cells from hair follicles as a cellular model for neurodevelopmental disorders. Stem Cell Res., 8: 134-140. DOI: 10.1016/j.scr.2011.09.003
Seifinejad, A., M. Tabebordbar, H. Baharvand, L. Boyer and G.H. Salakdeh, 2010. Progress and promise towards safe induced pluripotent stem cells for therapy. Stem Cell Rev. Rep., 6: 297-306. DOI: 10.1007/s12015-010-9121-X

Shi, Y., J.T. Do, C. Desponds, H.S. Hahm and H.R. Scholer et al., 2008. A combined chemical and genetic approach for the generation of induced pluripotent stem cells. Cell Stem Cell, 2: 525-528. DOI: $10.1016 /$ j.stem.2008.05.011

Sipp, D., 2010. Challenges in the clinical application of induced pluripotent stem cells. Stem Cell Res. Ther. DOI: $10.1186 /$ scrt9

Stadtfeld, M. and K. Hochedlinger, 2010. Induced pluripotency: History, mechanisms and applications. Genes Dev., 24: 2239-2263. DOI: $10.1101 / \operatorname{gad} .1963910$

Stadtfeld, M., K. Brennand and K. Hochedlinger, 2008. Reprogramming of pancreatic $\beta$ cells into induced pluripotent stem cells. Curr. Biol., 18: 890-894. DOI: 10.1016/j.cub.2008.05.010

Takahashi, K. and S. Yamanaka, 2006. Induction of pluripotent stem cells from mouse embryonic and adult fibroblast cultures by defined factors. Cell, 126: 663-676. DOI: 10.1016/j.cell.2006.07.024

Takenaka, C., N. Nishishita, N. Takada, L. Jakt and S. Kawamata, 2009. Effective generation of iPS cells from CD34+ cord blood cells by inhibition of p53. Exp. Hematol., 38: 154-162. DOI: 10.1016/j.exphem.2009.11.003

Tsai, S.Y., C. Clavel, S. Kim, Y.S. Ang and L. Grisanti et al., 2010. Oct4 and klf4 reprogram dermal papilla cells into induced pluripotent stem cells. Stem Cells. 28: 221-228. DOI: 10.1002/stem.281

Uemura, T., K. Takamatsu, M. Ikeda, M. Okada and K. Kazuki et al., 2012. Transplantation of induced pluripotent stem Cell-derived neurospheres for peripheral nerve repair. Biochem. Biophys. Res. Commun., 419: 130-135. DOI: 10.1016/j.bbrc.2012.01.154

Vitale, A.M., E. Wolvetang and A. Mackay-Sim, 2011. Induced pluripotent stem cells: A new technology to study human disease. Int. J. Biochem. Cell Biol., 43: 843-846. DOI: 10.1016/j.biocel.2011.03.013

Walia, B., N. Satija, R.P. Tripathi and G. Gangenahalli, 2012. Induced pluripotent stem cells: Fundamentals and applications of the reprogramming process and its ramifications on regenerative medicine. Stem Cell Rev. Rep., 8: 100-115. DOI: 10.1007/s12015-011-9279-X 
Woltjen, K., I.P. Michael, P. Mohseni, R. Desai and M. Mileikovskiet et al., 2009. Piggybac transposition reprograms fibroblasts to induced pluripotent stem cells. Nat., 458: 766-770. DOI: 10.1038/nature07863

Wong, G.K.Y. and A. Chiu, 2011. Gene therapy, gene targeting and induced pluripotent stem cells: Applications in monogenic disease treatment. Biotechnol. Adv., 29: 1-10. DOI: 10.1016/j.biotechadv.2010.07.005

Yakubov, E., G. Rechavi, S. Rozenblatt and D. Givol, 2010. Reprogramming of human fibroblasts to pluripotent stem cells using mRNA of four transcription factors. Biochem. Biophys. Res. Commun., 394: 189-193. DOI: 10.1016/j.bbrc.2010.02.150

Yamanaka, S., 2009. A fresh look at iPS cells. Cell, 137: 13-17. DOI: 10.1016/j.cell.2009.03.034
Yao, L., X. Yu, N. Hui and S. Liu, 2011. Application of iPS in assisted reproductive technology: Sperm from somatic Cells? Stem Cell Rev. Rep., 7: 714721. DOI: 10.1007/s12015-011-9236-8

Yu, J., K.F. Chau, M.A. Vodyanik, J. Jiang and Y. Jiang, 2011. Efficient feeder-free episomal reprogramming with small molecules. PLoS ONE, 6: e17557e17557, DOI: 10.1371/journal.pone.0017557

Zeng, F. and Q. Zhou, 2011. Induced pluripotent stem cells: On the road toward clinical applications. Stem Cell Regen. Med., 4: 427-438. DOI: 10.1007/978-160761-860-7-26

Zhou, H., S. Wu, J.Y. Joo, S. Zhu and D.W. Han et al., 2009. Generation of induced pluripotent stem cells using recombinant proteins. Cell Stem Cell, 4: 381-384. DOI: 10.1016/j.stem.2009.04.005 